\title{
Tumor suppressor and T-regulatory functions of Foxp3 are mediated through separate signaling pathways
}

\author{
EMIL HEINZE $^{1}$, GRACE CHAN ${ }^{2}$, RACHEL MORY $^{1}$, RAZ KHAVARI $^{1}$, ASIF ALAVI $^{1}$, SUE Y. CHUNG ${ }^{1}$, \\ ROBERT N. NISHIMURA ${ }^{3}$ and RICHARD H. WEISBART ${ }^{2}$ \\ ${ }^{1}$ Department of Medicine, Olive View-UCLA Medical Center, Sylmar, CA 91342; \\ ${ }^{2}$ Department of Research, Veterans Affairs Greater Los Angeles Healthcare System, Sepulveda, CA 91343; \\ ${ }^{3}$ Department of Neurology, University of California, Los Angeles, CA 90095, USA
}

Received December 16, 2010; Accepted May 11, 2011

DOI: $10.3892 / 01.2011 .307$

\begin{abstract}
Foxp3 is a nuclear transcription factor that is both a tumor suppressor factor and regulator of T-regulatory cell (Treg) function, and is a potential therapeutic target in both autoimmunity and cancer. In order to distinguish molecular pathways responsible for these separate Foxp3 functions, deletion mutants of Foxp3 proteins were transduced and analyzed for cytotoxic activity in human cancer cell lines Skov3, MDA-MB-231, MCF-7 and Jurkat. Human Foxp3 cDNA mutants were amplified and ligated to produce plasmids for direct cell transfection. Constructs were produced and confirmed by DNA sequencing. Lipofectamine 2000 was used for plasmid transfection. Foxp3 cells were then examined. The results of our experiments reveal retention of tumor suppressor function in the absence of NFAT binding and transcriptional activation required for Treg function. Our results have significant implications for the design of autoimmune and cancer therapies that target Foxp3 and Treg cells.
\end{abstract}

\section{Introduction}

Foxp3 is an X-linked nuclear transcription factor essential to the development and program of T-regulatory cells (Tregs) that protect against the development of autoimmunity $(1,2)$. The tumor suppressor activity of Foxp3 was identified when it was serendipitously observed that female mice heterozygous for the mutation in the X-linked scurfin gene (homolog to human Foxp3) have high rates of mammary cancer as well as other types of cancers (3). Additional studies confirmed a relationship between mutations of Foxp3 and breast cancer in

Correspondence to: Dr Richard Weisbart, Department of Research, Veterans Affairs Greater Los Angeles Healthcare System (111S), 16111 Plummer St., North Hills, CA 91343, USA

E-mail: rweisbar@ucla.edu

Abbreviations: Tregs, T-regulatory cells

Key words: transcription factors, apoptosis, immune regulation humans, and identified Foxp3-mediated repression of HER-2/ ErbB2 and SKP2 oncogenes as a potential mechanism for the tumor suppressor effect $(3,4)$. Although Tregs suppress the development of autoimmunity, they are thought to be permissive for the development of cancer $(5,6)$. Thus, Foxp3 has dual but opposing roles with respect to autoimmunity and cancer. Tregs and Foxp3 have become significant therapeutic targets in autoimmunity and cancer. Defining Foxp3 pathways responsible for its divergent functions should be helpful in the design of therapies that specifically target Foxp3. Development of the Treg function requires transcriptional activation and binding of Foxp3 to the transcription factor, NFAT (7). In this study, Foxp3 was found to exhibit tumor suppressor activity independent of transcriptional activation and binding NFAT.

\section{Materials and methods}

Cell lines. Human cancer cell lines Skov3 (ovarian), MDA-MB-231 (breast), MCF-7 (breast) and Jurkat (T cells) were obtained from the American Type Tissue Collection (Rockville, MD, USA).

Antibodies. The antibodies used included mouse monoclonal IgG1 to human Foxp3 (sc-56680) and mouse anti-goat IgG-HRP (sc-2354) (Santa Cruz Biotechnology, Santa Cruz, CA, USA).

Plasmid constructs. Human Foxp3 cDNA was amplified by PCR from plasmid 13250 (Addgene, Cambridge, MA, USA) and ligated into pIRES2-ZsGreen1 (Clontech, Mountain View, CA, USA) to produce the plasmid pIRES2-ZsGreen1-Foxp3, pIRES2-ZsGreen1-Foxp3C (C-terminal deletion of residues 328-431) and pIRES2-ZsGreen1-Foxp3N (N-terminal deletion of residues 1-196) for direct transfection into cancer cells (Fig. 1A). Molecular fusions of mAb $3 \mathrm{E} 10 \mathrm{Fv}$ were also generated as pPicZA-Fv-Foxp3, pPicZA-Fv-Foxp3WWRR (mutations T359W, N361W, E399 and E401R of Foxp3), pPicZA-Fv-Foxp3C (C-terminal deletion of residues 328-431) and pPicZA-Fv-Foxp3N (N-terminal deletion of residues 1-196) (Fig. 1A). Recombinant Foxp3 mutants were constructed using a QuikChange II site-directed mutagenesis 
kit (Stratagene, TX, USA). Constructs were confirmed by DNA sequencing.

Expression and purification of Foxp 3 and Fv-Foxp3 recombinant proteins. Recombinant proteins were expressed in X-33 cells, lysed by passage through a French Cell Press, and purified by immobilized metal ion affinity chromatography (IMAC) on Ni-NTA Agarose (Qiagen, Valencia, CA, USA) as previously described (8). Eluted protein was concentrated to $10 \mu \mathrm{g} / \mathrm{ml}$, reconstituted with fetal calf serum (FCS) to $5 \%$, and, the protein was exchanged dialyzed 100-fold in 30,000 MWCO spin filters (Millipore Corp., Billerica, MA, USA) against McCoy's medium (Mediatech, Inc., Herndon, VA, USA) containing 5\% glycerol.

Cytotoxicity of Foxp3 constructs in vitro. Foxp3 and Foxp3 mutant cDNA were ligated into the pIRES2-ZsGreen1 expression vector (Clontech, Mountain View, CA, USA) that co-expresses a green fluorescent protein with the cloned insert. Lipofectamine 2000 (Invitrogen, Carlsbad, CA, USA) was used according to the manufacturer's protocol for plasmid transfection into Skov-3 cells. Following transfection, Skov-3 cells were incubated for 7 days at $37^{\circ} \mathrm{C}$ in 24 -well plates. Fluorescent cells represent cells expressing Foxp3. Subsequently, fluorescent cells were examined for the number of dead vs. viable cells. One hundred transfected cells were counted in each of the triplicate wells, and the results were expressed as the mean percent \pm SD (standard deviation). In studies of antibody-mediated protein transduction, cancer cells in the medium containing 10\% FCS were added to each of the multiple wells in 96-well plates and grown to $40 \%$ confluence overnight in the presence of $5 \% \mathrm{CO}_{2}$. Fv-Foxp3 recombinant proteins were added undiluted $(100 \mathrm{nmol} / \mathrm{l})$ to duplicate wells and in serial dilutions with the X-33 lysate as a control. Cell death was measured by the nuclear uptake of propidium iodide, and the results were expressed as the mean percent \pm SD by counting 100 cells in 3 separate areas of duplicate wells.

Statistical analysis. A p-value was determined by a twotailed Student's t-test for non-paired samples assuming equal variances.

\section{Results}

Recombinant Fv-Foxp3 proteins. To determine whether Foxp3-mediated cytotoxicity requires NFAT, we produced and purified Foxp3 constructs shown to eliminate NFAT binding. In their study, Wu et al previously showed that the combination of selected mutations of Foxp3 C-terminal forkhead domain (FKH), T359W, N361W, E399 and E401R (WWRR) prevented NFAT binding (7). We produced Foxp3, Foxp3 WWRR mutant, and Foxp3 with C-terminal deletion of the entire FKH ( $\Delta$ aa 328-431) as molecular fusion proteins with mAb 3E10 cell-penetrating Fv fragment (Fig. 1A). The FKH is involved in DNA binding and nuclear localization, and enables transcriptional activation or repression (9). A Western blot analyis of the purified proteins developed with antibodies to Foxp3 showed recombinant proteins of the expected sizes (Fig. 1B).
A Foxp3 Constructs

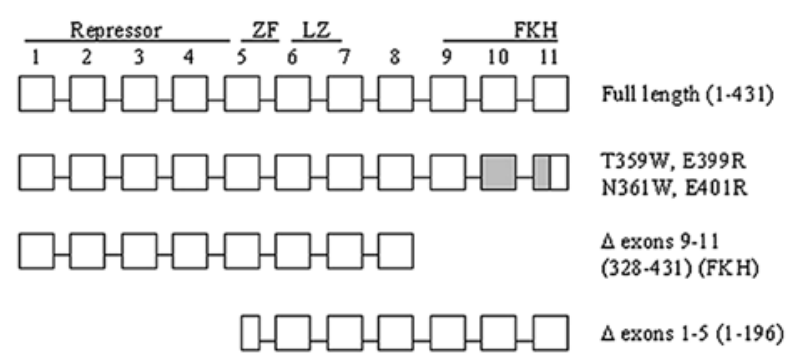

B

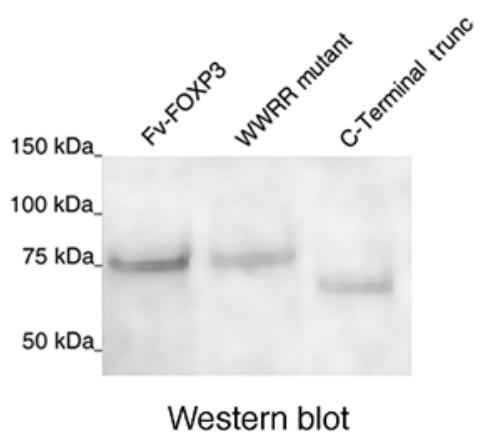

Figure 1. (A) Schematic representation of Foxp3 and mutant constructs (ZF, zinc finger; LZ, leucine zipper; and FKH, forkhead). Shaded areas show the location of mutations. (B) Western blotting of Fv-Foxp3 and Fv-Foxp3 mutant molecular fusion proteins developed with an antibody to the $\mathrm{N}$-terminus of human Foxp3.

Foxp3 cytotoxicity independent of NFAT. Cytotoxicity of Foxp3 in cancer cells was previously demonstrated by antibodymediated protein transduction $(8,10,11)$. In the present study, Fv-Foxp3 was cytotoxic in four cancer cell lines, but Foxp3 alone and the X-33 cell lysate as controls were not cytotoxic (Fig. 2). However, Fv-Foxp3 with WWRR mutations that prevent NFAT binding was also cytotoxic in these cancer cell lines (Fig. 2). Moreover, deletion of the entire Foxp3 FKH failed to eliminate Foxp3 cytotoxicity in cancer cells (Fig. 2). These results were confirmed by the direct transfection of Foxp3 cDNA into Skov-3 cells. Both full-length and C-truncated Foxp3 were cytotoxic (Fig. 3). However, an $\mathrm{N}$-terminal deletion mutant of Foxp3 (Fig. 1A) completely abrogated Foxp 3 cytotoxicity, indicating that the $\mathrm{N}$-terminus of Foxp3 is required for Foxp3 tumor suppressor activity (Fig. 3).

\section{Discussion}

Transcription factors form large complexes of interacting proteins that regulate gene activation pathways. Dissecting functional activation pathways is crucial to the design of therapeutic agents that target transcription factors. Tregs and Foxp3 have become significant therapeutic targets both in autoimmunity and cancer (13). However, inhibiting Treg function to enhance tumor rejection carries risks associated with developing autoimmunity. On the other hand, enhancing Treg function to control autoimmunity may predispose to the development of cancer. More studies are therefore required to better define the divergent signaling pathways of Foxp3, thereby aiding in the design of target-specific therapies. 

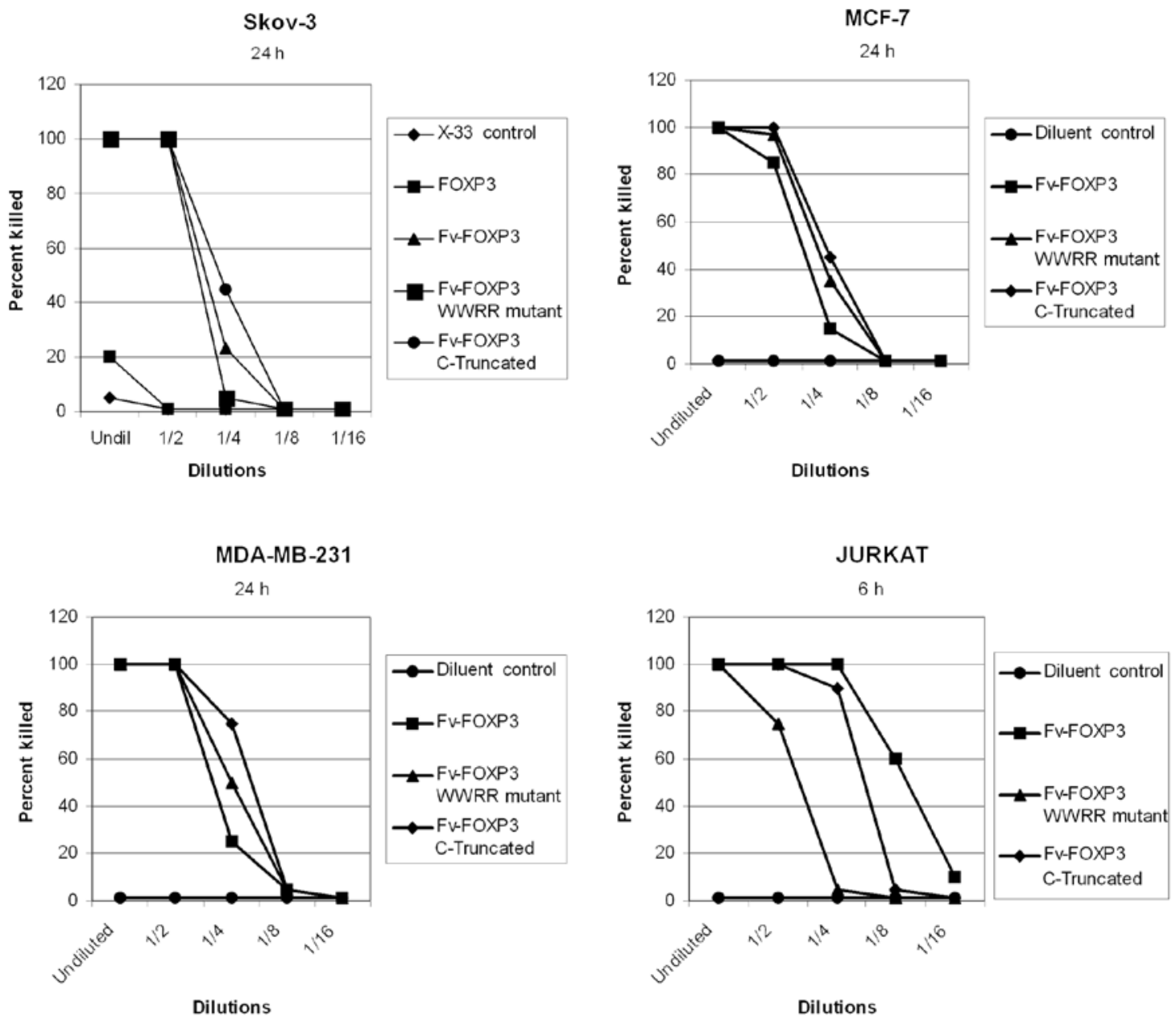

Figure 2. Effect of antibody-mediated transduction of Foxp3 and mutants on cytotoxicity of cancer cells. Dose-dependent killing of cancer cells by Fv-Foxp3, mutant Fv-Foxp3 WWRR (T359W, N361W, E399R and E401R) and C-truncated Fv-Foxp3 ( $\Delta$ aa 328-431). Fv-Foxp3 and mutants were incubated for $24 \mathrm{~h}$ with breast and ovarian cancer cell lines and $6 \mathrm{~h}$ with the Jurkat cells. Negative controls included the X-33 lysate and Foxp3 in Skov-3 cells and a diluent control in the remaining cell lines. Cell death was determined by the nuclear uptake of propidium iodide.

Effect of Foxp3 and mutant transfection on Skov-3 cells

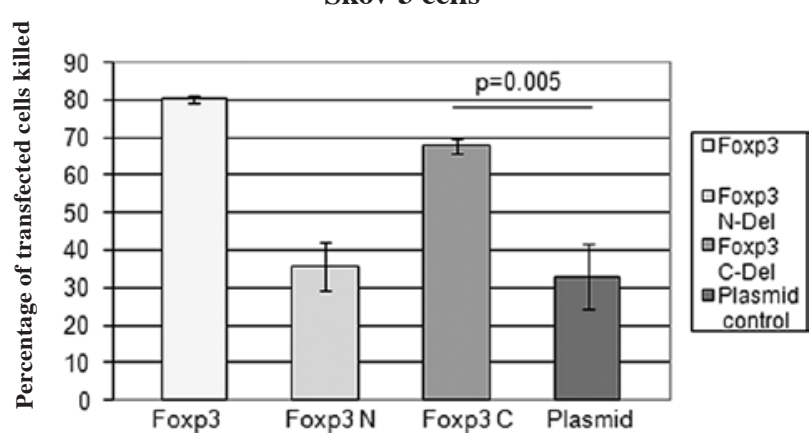

Figure 3. Effect of transfection of Foxp3 cDNA and mutants on the cytotoxicity of Skov-3 cells. Results are the percentage of transfected cells killed by Foxp3, Foxp3 C-terminal deletion mutant ( $\Delta$ aa 328-431) and Foxp3 $\mathrm{N}$-terminal deletion mutant ( $\Delta$ aa 1-196) compared to the empty plasmid vector. Transfected cells were identified by the presence of a green fluorescent protein. Results are expressed as the mean percentage killed $\pm \mathrm{SD}$, and are representative of two independent experiments.

We have investigated the use of a cell-penetrating antibody, $\mathrm{mAb} 3 \mathrm{E} 10$, and its Fv fragment as an intracellular and intra- nuclear delivery vehicle for the delivery of transcription factor proteins, such as p53 and Foxp3 $(8,12)$. The up-regulation of Foxp3 has been shown to be cytotoxic in cancer cells in vitro and in vivo $(3,8)$. However, it is of concern that up-regulating Foxp3 may enhance Treg function in vivo, thereby abrogating its clinical effectiveness as a tumor suppressor in vivo. The results of our study show that Foxp3 cytotoxicity can be separated from its effect on Treg cell function by eliminating NFAT binding. Although the repressor and FKHs of Foxp3 are critical for its Treg function (14), our study shows that cytotoxic activity is preserved in the absence of the FKH. Previous studies identified HER-2 and SKP2 oncogenes as potential targets suppressed by Foxp3 $(3,4)$. Our unexpected finding that the $\mathrm{N}$-terminal, but not the $\mathrm{C}$-terminal, deleted Foxp3 results in loss of cytotoxicity indicates that there may be additional mechanisms for Foxp3 cytotoxicity independent of the FKH. This is not unprecedented, as the p53 tumor suppressor has been shown to mediate apoptosis by both transcription-dependent and -independent pathways $(15,16)$. In our study, the role of Foxp3 N-terminus in cellular cytotoxicity may be related to its potential binding to a number of auxiliary transcription factors or chromatin-modifying proteins (17). Further studies are required to define the role of $\mathrm{N}$-terminal 
Foxp3 in cell toxicity. However, our finding that the Treg and tumor suppressor functions of Foxp3 are mediated through separate signaling pathways enables us to develop methods for the up-regulation of Foxp3-induced cancer cell cytotoxicity without enhancing Treg function.

\section{Acknowledgements}

This study was supported by a grant from the Veterans Affairs (RHW).

\section{References}

1. Hori S, Nomura T and Sakaguchi S: Control of regulatory T cell development by the transcription factor Foxp3. Science 299. 1030-1031, 2003.

2. Schubert LA, Jeffery E, Zhang Y, Ramsdell $\mathrm{F}$ and Ziegler SF: Scurfin (FOXP3) acts as a repressor of transcription and regulates T cell activation. J Biol Chem 276: 37672-37679, 2001.

3. Zuo T, Wang L, Morrison C, Chang X, Zhang H, Li W, Liu Y, Wang Y, Liu X, Chan MW, Liu JQ, Love R, Liu CG, Godfrey V, Shen R, Huang TH, Yang T, Park BK, Wang CY, Zheng P and Liu Y: FOXP3 is an X-linked breast cancer suppressor gene and an important repressor of HER-2/ErbB2 oncogene. Cell 129:1275-1286, 2007.

4. Zuo T, Liu R, Zhang H, Chang X, Liu Y, Wang L, Zheng P and Liu Y: FOXP3 is a novel transcriptional repressor for the breast cancer oncogene SKP2. J Clin Invest 117: 3765-3773, 2007.

5. Kim J, Lahl K, Hori S., Loddenkemper C, Chardhry A, deRoos P, Rudensky A and Sparwasser T: Depletion of Foxp ${ }^{3+}$ cells leads to induction of autoimmunity by specific ablation of regulatory $\mathrm{T}$ cells in genetically targeted mice. J Immunol 183: 7631-7634, 2009

6. Hinz S, Pagerols-Raluy L, Oberg H, Ammerpohl O, Grüssel S, Sipos B, Grützmann R, Pilarsky C, Ungefroren H, Saeger H, Klöppel G, Kabelitz D and Kalthoff H: Foxp3 expression in pancreatic carcinoma cells as a novel mechanism of immune evasion in cancer. Can Res 67: 8344-8350, 2007.
7. Wu Y, Borde M, Heissmeyer V, Feuerer M, Lapan AD, Stroud JC, Bates DL, Guo L, Han A, Ziegler SF, Mathis D, Benoist $\mathrm{C}$, Chen $\mathrm{L}$ and Rao A: FOXP3 controls regulatory $T$ cell function through cooperation with NFAT. Cell 126: 375-387, 2006.

8. Heinze E, Baldwin S, Chan G, Hansen J, Song J, Clements D, Aragon R, Nishimura R, Reeves M and Weisbart R: Antibodymediated protein therapy induces apoptosis in cancer cells in vitro and inhibits metastasis in vivo. Int J Oncol 35: 167-173, 2009.

9. Ziegler SF: FOXP3: Of mice and men. Annu Rev Immunol 24: 209-226, 2006.

10. Weisbart RH, Stempniak M, Harris S, Zack DJ and Ferreri K: An autoantibody is modified for use as a delivery system to target the cell nucleus: Therapeutic implications. J Autoimmun 11: 539-546, 1998.

11. Hansen JE, Tse CM, Chan G, Heinze ER, Nishimura RN and Weisbart RH: Intranuclear protein transduction through a nucleoside salvage pathway. J Biol Chem 282: 20790-20793, 2007.

12. Hansen JE, Fischer LK, Chan G, Chang SS, Baldwin SW, Aragon RJ, Carter JJ, Lilly M, Nishimura RN, Reeves ME and Weisbart RH: Antibody-mediated p53 protein therapy prevents liver metastasis in vivo. Cancer Res 67: 1769-1774, 2007.

13. Katoh H, Zheng P and Liu Y: Signalling through FOXP3 as an X-Linked tumor suppressor. Int J Biochem Cell Biol 42: 1784-1787, 2010

14. Lopes JE, Torgerson TR, Schubert LA, Anover SD, Ocheltree EL, Ochs HD and Ziegler SF: Analysis of FOXP2 reveals multiple domains required for its function as a transcriptional repressor. J Immunol 177: 3133-3142, 2006.

15. Matas D, Sigal A, Stambolsky P, Milyavsky M, Weisz L, Schwartz D, Goldfinger $\mathrm{N}$ and Rotter V: Integrity of the $\mathrm{N}$-terminal transcription domain of $\mathrm{p} 53$ is required for mutant p53 interference with drug-induced apoptosis. EMBO J 20: 4163-4172, 2001.

16. Haupt S, Berger M, Goldberg Z and Haupt Y: Apoptosis - the p53 network. J Cell Sci 116: 4077-4085, 2003

17. Zhou Z, Song X, Li B and Greene MI: FOXP3 and its partners: structural and biochemical insights into the regulation of FOXP3 activity. Immunol Res 42: 19-28, 2008. 